\title{
Ischemia, Cerebrovascular Diseases. Their Characteristics In Children
}

\author{
Kazakov Khabibulla Abdullayevich \\ Assistant of the Department of Propaedeutics of Internal Professions, Urgench branch of the \\ Tashkent Medical Academy, Urgench, Uzbekistan. \\ Satlikov Rashid Karimovich \\ Candidate of Medical Sciences, Department of Propaedeutics of Internal Professions, \\ Urgench branch of the Tashkent Medical Academy, Urgench, Uzbekistan. \\ Khaitbayev Jo'rabek Azatboyevich \\ Department of Propaedeutics of Internal Professions, Urgench branch of the Tashkent \\ Medical Academy, Urgench, Uzbekistan.
}

Journal Website: http://usajournalshub.c om/index,php/TAJMSP $\mathrm{R}$

Copyright: Original content from this work may be used under the terms of the creative commons attributes 4.0 licence.

\section{ABSTRACT}

In this article you can get a brief information about cardiovascular diseases, the process of their occurrence, the order of circulatory disorders, the effect of hormones produced by the kidneys.

\section{KEYWORDS}

Hypertension, angiotensin 1, Renin-angiotensin system, arteriola.

\section{INTRODUCTION}

Hypertension - (essential hypertension, primary arterial hypertension) - is a chronic pain, the main clinical symptom of which is a prolonged and persistently high blood pressure. The disease is observed in middle age in women and after 50 years in men. Primary arterial hypertension accounts for $90 \%$ of hypertension and symptomatic hypertension for 10\%. They are safe (good quality) and dangerous (bad quality). Specific features: 1) widespread 2) long-term asymptomatic 3) causing life-threatening complications.

Blood pressure and heart rhythms are controlled by the neurohumoral pathway (synonyms of baroreceptors mechanoreceptors, pressoreceptors, interoreceptor pressure-sensitive cells).

Mental-emotional disturbances lead to functional disorders of the vascular center and 
the involvement of reflex and humoral factors in the mechanism of disease pathogenesis. Blood pressure depends on the amount of ore being pumped from the heart into the arteries, the resistance of the peripheral arteries. These are controlled by nervous, baroreceptor, chemoreceptor systems, humoral factors. Renal excretory mechanisms also play an important role in the control of blood pressure.

Among the reflex factors are changes in the depressant effects of receptors on the carotid sinuses and aortic arch (atrophic changes in the carotid sinus), as well as activation of the sympathetic nervous system.

Renin-angiotensin system: YuGA cells are hyperplasia, nucleus, interstitial cells are atrophied, under hypoxia, cells of the upper staglomerular complex produce renin, in the serum it is converted to the liver in the form of decapeptide angiotensin 1 and angiotensin. Angiotensin $P$ narrows blood vessels, increases aldosterone secretion, aldosterone in turn affects the reabsorption of sodium and water in the renal tubules, prepares the ground for an increase in vasoconstrictor effect, blood pressure is raised. Sodium and water retention in the body increases the volume and peripheral resistance of the heart, increases the sensitivity of smooth muscle cells to vasoactive inhibitors.

The vascular center 1) reflex (sinuses, symptomatic nervous system is activated. hormonal (pituitary - diencephalic area pressor effect is increased. Anterior and posterior pituitary stem cells are hyperplasia. 2) adrenal gland hyperplasia, catecholamines are produced.

With the development of the disease, a ring is formed in the presence of humoral factors: narrowing of the renal arteries (vasoconstriction, arteriosclerosis) hypersecretion of renin - increased production of angiotensin - aldosterion hypersecretion sodium retention - increased sensitivity of arteriolar walls to pressure factors

\section{ETIOLOGY OF HYPERTENSION}

In the etiology of hypertension, the central nervous system is of paramount importance. (G.F. Lang). Exposure to the central nervous system leads to disruption of vascular tone nerve regulation and the onset of spasms. The pathogenesis of hypertension is thought to be related to impaired central nerve regulation of the peripheral circulation. As a result of the neuro-psychiatric stress on the cerebral cortex, this control is disrupted, resulting in the formation of ammunition.

After spasm in the arterioles, structural changes occur, however, the renal vessels are particularly damaged. Insufficient supply of ore to the kidney (ischemia) leads to the accumulation of pressor substances in the deposit. The endocrine glands also undergo changes during the course of the disease. Of particular importance is the increase in basophils in the anterior pituitary gland, which also produces pressor substances. Decreased levels of estrogen and androgens during a decrease in the activity of the gonads, increase the function of the cells of the adrenal cortex, lead to the formation of large amounts of catecholamines, adrenaline. It is these humoral and endocrine factors that seem to strengthen hypertension and cause high blood pressure over time.

According to membrane theory, in the genesis of essential hypertension, the genetic defect in the cell membrane that controls the distribution of calcium within the cell is the primary link. It alters the contractile properties of vascular smooth muscle, enhances the release of mediators at nerve endings, increases the activity of peripheral sections of 
the sympathetic nervous system, resulting in narrowing of arterioles and arterial hypertension.

Decreased magnesium content increases the sensitivity of the vascular wall to pressor effects, alters the sensitivity of the sympathetic nervous system centers to stressors, leading to a decrease in the vasodilating effect.

Golikov A.P. classified hemodynamic changes in arterial hypertension into types:

1. Hyperkinetic (increased heart rate per minute) - peripheral resistance is normal).

2. Hypokinetic (decreased heart rate) peripheral resistance increases sharply).

3. Eukinetic (heart rate does not change) peripheral resistance is increased.

\section{PATHOLOGICAL}

ANATOMY

OF

\section{HYPERTENSIVE DISEASE}

Injury to the small arteries and arterioles is typical for arterial hypertension, with arteriosclerosis, hyalinosis, or fibrinoid necrosis. Elastofibrosis and atherosclerosis are characteristic of elastic and muscular-elastic arteries.

There are three stages in the development of the disease.

1. Functional period

2. The period of morphological changes in the arteries with arterioles

3. The period when secondary morphological changes occur in the organs due to vascular changes.

1) Functional period - arterial mining pressure rises (transient hypertension) under the influence of emotion.

Muscle tone of arterioles increases, hypertrophy and elastosis of arterioles and small arteries in the muscular layer (the inner elastic membrane of vessels divides into several layers, enters the subintimal cavity and sticks, new elastic fibers are formed, resulting in muscular arteries, elastic-type arteries) left heart rate increases due to the need to overcome the flow through the arterioles, which reduce the flow. Therefore, left ventricular hypertrophy is established in the earliest stages of hypertension. These changes are constructed as compensatory adaptive reactions directed at increasing pressure within the vessels. The functional period lasts from a few months to 1-2 years.

2) blood pressure rises for a long time (stable), the period of morphological (increased alteration processes in the walls) arterioles and arteries is characterized by changes in the activity of the cardiovascular system.

In the second stage of the disease it is found that endothelial barrier permeability is increased and the plasma proteins penetrate the arteriole wall.

This process of plasma infiltration in the arterioles is very close to the fibrinoid bending that occurs in the presence of fibrin. The elements that make up the artery wall (muscle and elastic fibers) can undergo necrosis (fibrinoid necrosis). In this case, the path of the arterioles can be completely narrowed and the wall can be completely blocked. The endothelium collapses and dies.

The arterioles of the kidneys, brain, adrenal glands, pancreas and peritoneum, pancreas, gastrointestinal tract and other organs undergo major changes (hyalinosis of arteries and small arterioles).

When the walls of arterioles are deeply destroyed and necrotic, fat droplets in the plasma masses, phagocytizing lipids around the walls and sides of the altered arterioles, turn into xanthoma cells. Atherosclerotic changes develop circularly in the wall of 
muscle-type arteries, which further narrows the vascular space (especially when the damage to the coronary arteries is more severe).

In other cases, the protein masses in the walls of the arterioles gradually accumulate, as a result of spastic (contraction) processes in the arterioles break down the elastic fibers and connective tissue grows between the muscle fibers (elastofibrosis).

This indicates that during the second period of hypertension, the rest is suppressed and the rest is followed again, or in other words, there are crises of hypertension. Crisis can be clinically and morphologically accompanied by damage to the renal arterioles, as well as damage to the arterioles of the brain, intestines or gallbladder.

Plasma infiltration in the walls of the capillaries of the brain, myocardium, kidneys, fibrinoid necrosis occurs, xanthoma cells appear, hyalinosis is found in the walls of the capillaries.

\section{CONCLUSION}

When blood pressure is elevated, the left ventricle of the heart becomes enlarged and hypertrophic due to changes in the peripheral vascular bed, which is compensatory in nature. The heart weighs 500-900 grams, and the thickness of the left ventricle of the heart reaches $2-3 \mathrm{~cm}$ when measured from the base of the heart, the heart cavities dilate, muscle fibers are wide, nuclei are enlarged, organoids and organelles are hypertrophied and hyperplasia (concentric enlargement). Increased hypoxia leads to myocardial dystrophy and necrosis, dilatation of the left ventricular cavity (eccentric hypertrophy), diffuse capillary cardiosclerosis in the myocardium, increased atherosclerotic changes in the coronary arteries and decompensation of cardiac activity (hypertrophy of the right ventricular muscles, enlargement of the left ventricle, thinning of the wall is observed.)

\section{REFERENCES}

1. M.S. Abdullaxujaeva. Fundamentals of human pathology. Tashkent. Medical publication named after Abu Ali Ibn Sina. 1998. P-section. 82-93 p.

2. Pathological anatomy (lecture course). P/ r. academician RAMN V.V.Serova, academician RAN and RAMN M.A.Paltseva. M.Moskva.1998. 225-236 p.

3. A.I.Strukov et al. Pathological anatomy. $M$ .: Med. 1985. 282-296 p.

4. VA Alimov, ZZ Egamberdieva: Application of pathological anatomy Tashkent. Medical publication named after Abu Ali Ibn Sina. 1993. 80-85 p.

5. Computer test control programs on the subject of pathological anatomy. 1TashDavTI (Department of Pathological Anatomy). 2002.

6. Ya.Yarygin Atlas patologicheskoy anatomii. M .: Medicine. 1986. 229-248S.

7. V.V.Serov et al. Guidance to practical training in pathological anatomy. $M$.: Med., 1987. 124-138 p. 